\title{
Article
}

\section{On parametric equivalent, isomorphic and unique sets}

\author{
J. Kok ${ }^{1,2, *}$ and J. Shiny ${ }^{3}$ \\ 1 Independent Mathematics Researcher, City of Tshwane, South Africa. \\ 2 Visiting Faculty at CHRIST (Deemed to be a University), Bangalore, India. \\ 3 Mathematics Research Center, Mary Matha Arts and Science College, Kerala, India. \\ * Correspondence: jacotype@gmail.com; johan.kok@christuniversity.in; Tel.: +27646547285 \\ Communicated by: Muhammad Kamran Jamil \\ Received: 20 January 2021; Accepted: 19 March 2021; Published: 28 March 2021.
}

\begin{abstract}
This short paper introduces the notions of parametric equivalence, isomorphism and uniqueness in graphs. Results for paths, cycles and certain categories (or types) of trees with regards to minimum confluence sets are presented.
\end{abstract}

Keywords: Parametric equivalence; Parametric isomorphism; Parametric uniqueness.

MSC: 05C07; 05C12; 05C15; 05C38.

\section{Introduction}

C oncepts, notation and graph parameters without formal definitions can be clarified in [1-3]. Unless stated otherwise, graphs will be finite, undirected and connected simple graphs. A shortest path having end vertices $u$ and $v$ is denoted by $u-v_{(i n G)}$. If $d_{G}(u, v) \geq 2$ then a vertex $w$ on $u-v_{(i n G)}, w \neq u$, $w \neq v$ is called an internal vertex on $u-v_{(i n G)}$. When the context is clear the notation such as $d_{G}(u, v), \operatorname{deg}_{G}(v)$ can be abbreviated to $d(u, v), \operatorname{deg}(v)$ and so on.

Numerous graph or element parameters have been studied over years. These parameters can be categorized into two main groups. These are (i) graph structural properties such as, vertex degree, open and closed neighborhoods, graph diameter, connectivity, independence, domination and so on and (ii) derivative parameters stemming from a variety of vertex and/or edge labeling regimes or measure conditions. The latter is the study of the existence of vertex and/or edge subsets which establish graphical compliance with the definition of the stated labeling regime or measure condition.

Let $\rho$ denote some minimum or maximum graph parameter related to subsets $V(G)$ of graph $G$. A minimum dominating set $X \subset V(G)$ (therefore $\rho=\gamma(G)=|X|$ ) serves as an example. Let $X, Y$ be distinct subsets of $V(G)$ which satisfy $\rho$. Then $X$ and $Y$ is said to be parametric equivalent or $\rho$-equivalent denoted by, $X \equiv_{\rho} Y$. Furthermore, if $X \equiv_{\rho} Y$ and the induced graphs $\langle V(G) \backslash X\rangle,\langle V(G) \backslash Y\rangle$ are isomorphic then $X$ and $Y$ are said to be parametric isomorphic. This isomorphic relation is denoted by $X \cong \cong_{\rho} Y$. Let all the vertex subsets of graph $G$ which satisfy $\rho$ be $X_{1}, X_{2}, X_{3}, \ldots, X_{k}$. If $X_{1} \cong_{\rho} X_{2} \cong_{\rho} X_{3} \cong_{\rho} \ldots \cong_{\rho} X_{k}$ then $X_{i}, 1 \leq i \leq k$ are said to be parametric unique or $\rho$-unique. The graph $G$ is said to have a parametric unique or $\rho$-unique solution (or parametric unique $\rho$-set). An interesting interpretation is that if $G$ has a unique (exactly one) $\rho$-set $X$, then $X$ is a parametric unique $\rho$-set. The converse does not necessarily hold true. The notion of uniqueness has now been generalized for graph parameter studies.

The motivation is that in many real life application the choice between $\rho$-sets are often subject to additional conditions such as centrality, accessibility, domination, conflict with regards to transportation or data flow or clustering within social networks.

\section{Confluence in graphs}

The notion of a confluence set (a subset of vertices) of a graph $G$ was introduced in [4]. For a non-complete graph $G$, a non-empty subset $\mathcal{X} \subseteq V(G)$ is said to be a confluence set if for every unordered pair $\{u, v\}$ of distinct vertices (if such exist) in $V(G) \backslash \mathcal{X}$ for which $d_{G}(u, v) \geq 2$ there exists at least one $u-v_{(i n G)}$ with at 
least one internal vertex, $w \in \mathcal{X}$. Also any vertex $u \in \mathcal{X}$ is called a confluence vertex of $G$. A minimal confluence set $\mathcal{X}$ (also called a $\zeta$-set) has no proper subset which is a confluence set of $G$. The cardinality of a minimum confluence set is called the confluence number of $G$ and is denoted by $\zeta(G)$. A minimal confluence set is denoted by $\mathcal{C}$. To distinguish between different graphs the notation $\mathcal{C}_{G}$ may be used for a minimum confluence set of G. Recall two important results from [4].

Theorem 1. [4] For a path $P_{n}, n \geq 1$,

$$
\zeta\left(P_{n}\right)= \begin{cases}0, & \text { if } n=1 \text { or } 2 \\ \left\lfloor\frac{n}{3}\right\rfloor, & \text { if } n \geq 3\end{cases}
$$

Theorem 2. [4] For a cycle $C_{n}, n \geq 3$,

$$
\zeta\left(C_{n}\right)= \begin{cases}0, & \text { if } n=3 \\ 1, & \text { if } n=4 \\ \left\lceil\frac{n}{3}\right\rceil, & \text { if } n \geq 5\end{cases}
$$

The path $P_{3}=v_{1} v_{2} v_{3}$ has confluence sets $X_{1}=\left\{v_{1}\right\}, X_{2}=\left\{v_{2}\right\}, X_{3}=\left\{v_{3}\right\}$. Hence, $X_{1} \equiv_{\zeta} X_{2} \equiv_{\zeta} X_{3}$. Clearly, $X_{1} \cong_{\zeta} X_{3}$ because $\left\langle\left(V\left(P_{3}\right) \backslash\left\{v_{1}\right\}\right\rangle \cong\left\langle V\left(P_{3}\right) \backslash\left\{v_{3}\right\}\right\rangle\right.$. However, since $X_{1} ¥_{\zeta} X_{2} \oiint_{\zeta} X_{3}$ the path $P_{3}$ does not have a parametric unique $\zeta$-set. Similar reasoning shows that path $P_{5}=v_{1} v_{2} v_{3} v_{4} v_{5}$ has a parametric unique $\zeta$-set. From [5] it is known that a path $P_{n}, n=5+3 i, i=0,1,2, \ldots$ has a unique $\zeta$-set. Therefore, it has a parametric unique $\zeta$-set.

Proposition 1. A path $P_{n}$ has a parametric unique $\zeta$-set if and only if $n=1,2$ or $n=4+3 i$ or $n=5+3 i$, $i=0,1,2, \ldots$.

Proof. Part 1. The cases $n=1,2$ follow from the fact that both $P_{1}, P_{2}$ are complete. Thus $\mathcal{C}_{P_{1}}=\mathcal{C}_{P_{2}}=\varnothing$ and is respectively, unique. Therefore parametric unique.

For $n=4+3 i, i=0,1,2, \ldots$, the result follows from the fact that, without loss of generality, the $\zeta$-sets $X_{1}=\left\{v_{3}, v_{6}, v_{9}, \ldots, v_{n-1}\right\}, X_{2}=\left\{v_{3}, v_{6}, v_{9}, \ldots, v_{n-4}, v_{n-2}\right\}, X_{3}=\left\{v_{3}, v_{6}, v_{9}, \ldots v_{n-7}, v_{n-5}, v_{n-2}\right\}$ and so on through back stepping until $X_{\zeta\left(P_{n}\right)+1}=\left\{v_{2}, v_{5}, v_{8}, \ldots v_{n-8}, v_{n-5}, v_{n-2}\right\}$, are all parametric isomorphic.

For $n=5+3 i, i=0,1,2, \ldots$, the result follows from the fact that $P_{n}$ has a unique $\zeta$-set [5].

Part 2. If $P_{n}$ has a parametric unique $\zeta$-set we use elimination through induction to prove the converse. It is easy to verify that $P_{n}$ could be $P_{1}$ or $P_{2}$. For more valid converse options it is easy to verify that $P_{n}$ could be $n=4+3 i, n=5+3 i, i=0,1,2, \ldots$. It is easy to verify that $P_{3}$ does not have a parametric unique $\zeta$-set. For $P_{6}, \zeta\left(P_{6}\right)=2$. Obviously and amongst others, the sets $X_{1}=\left\{v_{3}, v_{5}\right\}$ and $X_{2}=\left\{v_{3}, v_{6}\right\}$ are $\zeta$-sets. Since, $X_{1} \varpi_{\zeta} X_{2}$ the converse does not hold for $P_{6}$. Through immediate induction it follows that the converse does not hold for $P_{3 i}, i=2, \ldots$ Since, $\mathbb{N} \backslash\{x \in \mathbb{N}: x=3 i, i=1,2, \ldots\}=\{y \in \mathbb{N}: y=4+3 i$, $i=0,1,2, \ldots\} \cup\{y \in \mathbb{N}: y=5+3 i, i=0,1,2, \ldots\} \cup\{1,2\}$ the converse follows.

Let a cycle $C_{n}, n \geq 3$ have vertex set $V\left(C_{n}\right)=\left\{v_{i}: i=1,2,3, \ldots, v_{n}\right\}$ and edge set $E\left(C_{n}\right)=\left\{v_{i} v_{i+1}: 1 \leq\right.$ $i \leq n-1\} \cup\left\{v_{1} v_{n}\right\}$.

Proposition 2. A cycle $C_{n}$ has a parametric unique $\zeta$-set if and only if $n=3,4$ or $n=5+3 i$ or $n=6+3 i$, $i=0,1,2, \ldots$

Proof. Part 1. The case $n=3$ follows from the fact that $C_{3}$ is complete. For $n=4$ we have $\left\{v_{i}\right\}, 1 \leq i \leq 4$ a $\zeta$-set and $\left\{v_{i}\right\} \cong_{\zeta}\left\{v_{j}\right\}$.

For $n=5+3 i, i=0,1,2, \ldots$, a valid minimum confluence set selection procedure is as follows. Let $X_{1}=\left\{v_{1}, v_{4}, v_{7}, \ldots, v_{n-1}\right\}$. It is easy to verify without loss of generality, that with $v_{1}$ the initiation vertex the procedure yields a unique $\zeta$-set. Applying the procedure through consecutive modular counting for each $v_{i}$, $1 \leq i \leq n$ yields unique sets $X_{i}$ in respect of the initiation vertex $v_{i}$. For each set $X_{i}$ it follows that

$$
\left\langle V\left(C_{n}\right) \backslash X_{i}\right\rangle=\underbrace{P_{2} \cup P_{2} \cup \cdots \cup P_{2}}_{\left\lfloor\frac{n}{3}\right\rfloor \text { times }} \cup P_{1}=\left\lfloor\frac{n}{3}\right\rfloor P_{2} \cup P_{1} .
$$


Hence, $X_{i} \cong_{\zeta} X_{j}$ for all pairs of $\zeta$-sets. In fact the modular counting results in some identical $\zeta$-sets which need not be replicated. We conclude that cycles $C_{n}, n=5+3 i, i=0,1,2, \ldots$ have parametric unique $\zeta$-sets.

For $n=6+3 i, i=0,1,2, \ldots$ the reasoning is similar.

Part 2. If $C_{n}$ has a parametric unique $\zeta$-set we use elimination through induction to prove the converse. It is easy to verify that $C_{n}$ could be $C_{3}$ or $C_{4}$. For more valid converse options it is easy to verify that $C_{n}$ could be $n=5+3 i, i=0,1,2, \ldots$ or $n=6+3 i, i=0,1,2, \ldots$ For $C_{n}, n=7+3 i, i=0,1,2, \ldots$ we note that $C_{7}$ has at least the $\zeta$-sets, $X_{1}=\left\{v_{1}, v_{4}, v_{7}\right\}$ and $X_{2}=\left\{v_{1}, v_{4}, v_{6}\right\}$. Also, $\left\langle V\left(C_{7}\right) \backslash X_{1}\right\rangle \nsubseteq\left\langle V\left(C_{7}\right) \backslash X_{2}\right\rangle$ hence, $X_{1} \neq_{\zeta} X_{2}$. Therefore the converse does not hold for $C_{7}$. Through immediate induction it follows that the converse does not hold for $C_{(7+3 i)}, i=0,1,2, \ldots$ Since, $\mathbb{N}_{\geq 3} \backslash\{x \in \mathbb{N}: x=7+3 i, i=0,1,2, \ldots\}=\{y \in \mathbb{N}: y=5+3 i$, $i=0,1,2, \ldots\} \cup\{z \in \mathbb{N}: z=6+3 i, i=0,1,2, \ldots\} \cup\{3,4\}$ the converse follows.

Corollary 1. For a cycle $C_{n}$ which has a parametric unique $\zeta$-set and with regards to vertex labeling let $t$ be the number of parametric isomorphic $\zeta$-sets which originates from $v_{i} \in V\left(C_{n}\right)$. Then $C_{n}$ has $\kappa\left(C_{n}\right)=\frac{n t}{\zeta\left(C_{n}\right)}$ distinct parametric isomorphic $\zeta$-sets. This implies that

(a) If $C_{n}, n=6+3 i, i=0,1,2, \ldots$ then $\kappa\left(C_{n}\right)=\frac{n}{\zeta\left(C_{n}\right)}$.

(b) If $C_{n}, n=5+3 i, i=0,1,2, \ldots$ then $\kappa\left(C_{n}\right)=n$.

Proof. The claim $\kappa\left(C_{n}\right)=\frac{n t}{\zeta\left(C_{n}\right)}$ is trivial.

(a) Let $n=6+3 i, i=0,1,2, \ldots$ : Thus $\zeta\left(C_{n}\right) \geq 2$. From the proof of Proposition 2(Part 1) it follows that a vertex $v_{i} \in V\left(C_{n}\right)$ yields exactly one $\zeta$-set. Hence, $t=1$ implying that any two vertices in a $\zeta$-set initiate identical $\zeta$-sets. The aforesaid settles the result.

(b) Let $n=5+3 i, i=0,1,2, \ldots$ : A valid minimum confluence set selection procedure is as follows. Let $X_{1}=\left\{v_{1}, v_{4}, v_{7}, \ldots, v_{n-1}\right\}$. It follows that $X_{2}=\left\{v_{1}, v_{4}, v_{7}, \ldots, v_{n-2}\right\}$ is valid. By similar modular back shifting it follows that $X_{3}=\left\{v_{1}, v_{4}, v_{7}, \ldots, v_{n-5}, v_{n-2}\right\}$ is valid and so on. Hence, vertex $v_{1}$ initiates exactly $\zeta\left(C_{n}\right)$ parametric isomorphic $\zeta$-sets. Therefore, $\kappa\left(C_{n}\right)=\frac{n \zeta\left(C_{n}\right)}{\zeta\left(C_{n}\right.}=n$.

\section{Types of trees}

The authors are not aware of a unified classification of trees. The classification below is not a partition hence some categories (or families) are sub-categories of others. It is merely the specialization of structure which motivates the classification. When $k, k \geq 1$ leafs (or pendent vertices) are attached to a selected vertex $v$ it is said that a $k$-bunch of leafs has attached to $v$.

(a) Paths is a tree with exactly two leafs.

(b) A star $S_{1, n}, n \geq 3$ (sub-category of spiders) has a central vertex $v_{0}$ with $n$ leafs.

(c) A $\ell$-star $S_{\ell, n \times m}, \ell \geq 2, n, m \geq 2$ has a path $P_{\ell}=v_{1} v_{2} v_{3} \cdots v_{\ell}$ with a $n$-bunch of leafs attached to say, $v_{1}$ and a $m$-bunch of leafs attached to $v_{\ell}$.

(d) A spider $S_{n}^{*}, n \geq 3$ is a starlike tree with one vertex $v_{0}$ of degree $n$ and all other vertices have degree at most 2. Clearly, $S_{n}^{*}, n \geq 3$ has $n$ pendent vertices. Hence in this context $n$ does not mean the order of a spider. Put differently, a spider has a central vertex $v_{0}$ which is attached with an edge to exactly one end-vertex of each path $P_{m_{1}}, P_{m_{2}}, \ldots, P_{m_{n}}, n \geq 3$.

(e) A caterpillar $C_{n_{1} \star n_{2} \star \cdots, \cdots n_{k}}, n_{i}, k \geq 1$ has a central path (or spine) $P_{m}, m \geq \max \{3, k\}$ with each $n_{i}$-bunch of leafs, $i=1,2,3, \ldots, k$ attached to a distinct vertex of $P_{m}$. If all $n_{i}=1$ a trivial lobster is obtained.

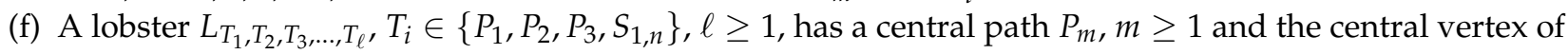
each $T_{i}$ be it an isolated vertex for $P_{1}$ or an end-vertex for $P_{2}$ or $v_{2}$ for $P_{3}$ or $v_{0}$ for stars are connected by an edge to some vertex of $P_{m}$. Hence a vertex of $T_{i}$ is within distance 2 from some vertex of $P_{m}$. A lobster has the property that if all leafs are removed a caterpillar is obtained.

(g) A finite $(k, d)$-regular tree $T_{k, d}, d \geq 3, k \geq 1$ are obtained as follows: Take a central vertex $v_{0},(k=0)$ and attach $d$ leafs to $v_{0}\left(1^{\text {st }}\right.$-iteration $\left.k=1\right)$, then for each new leaf attach $d-1$ leafs $\left(2^{n d}\right.$-iteration $\left.k=2\right)$, then for each new leaf attach $d-1$ leafs, $\cdots$, until the $k^{\text {th }}$-iteration. Note that for a $(k, d)$-regular tree $T_{k, d}, d \geq 3, k \geq 1$ each leaf is an end-vertex of some diam-path and $\operatorname{diam}\left(T_{k, d}\right)=2 k$ and $v_{0}$ is on every diam-path. 
(h) All other trees not in (a) through to (g).

Recall that the pendent degree of vertex $u \in V(G)$ denoted by $\operatorname{deg}_{p}(u)$ be the number of leafs adjacent to $u$. The open and closed pendent neighborhood of a vertex $v$ are respectively, $N_{p}(v)=\{$ leafs of $v\}$ and $N_{p}[v]=N_{p}(v) \cup\{v\}$. Also, a vertex $v$ to which a leaf $u$ is attached is called the pre-leaf of $u$ or simply, pre-leaf $v$. For paths the result with regards to parametric uniqueness is known (Proposition 1). A star has the unique $\zeta$-set, $\left\{v_{0}\right\}$ which implies it has a parametric unique $\zeta$-set.

Proposition 3. A $\ell$-star $S_{\ell, n * m}, \ell \geq 2, n, m \geq 2$ has a parametric unique $\zeta$-set if and only if $P_{\ell-2}=v_{2} v_{3} v_{4} \cdots v_{\ell-1}$, has $\ell-2=4+3 i$ or $\ell-2=5+3 i, i=0,1,2, \ldots$.

Proof. If $\ell=2$, then $\ell-2=0$, thus $P_{\ell-2}=\varnothing$. For both $\ell=3,4$ the paths $P_{1}, P_{2}$ are complete. For $\ell-2=4+3 i$ or $\ell-2=5+3 i, i=0,1,2, \ldots$, the result is a direct consequence of the proof found in Proposition 1 and the fact that both $\left\langle N_{p}\left[v_{2}\right]\right\rangle,\left\langle N_{p}\left[v_{\ell-1}\right]\right\rangle$ are stars.

Proposition 4. A spider $S_{n}^{*}, n \geq 3$ has a parametric unique $\zeta$-set if and only if, either (a) each $P_{m_{i}}, 1 \leq i \leq n$ has a parametric $\zeta$-set or (b) each $P_{m_{i}}, m_{i}=3 j, j=1,2,3, \ldots$ Furthermore,

(a) $\zeta\left(S_{n}^{*}\right)=1+\sum_{i=1}^{n} \zeta\left(P_{m_{i}}\right)$.

(b) $\zeta\left(S_{n}^{*}\right)=\sum_{i=1}^{n} \zeta\left(P_{m_{i}}\right), m_{i}=3 j, j=1,2,3, \ldots$

Proof. It is known that a leaf need not be in a $\zeta$-set of any graph. See Lemma 8 in [5]. From the proof of Proposition 1 it follows that if a path has a parametric unique $\zeta$-set, an end-vertex cannot be in such set. Therefore $v_{0}$ is in all $\zeta$-sets of $S_{n}^{*}$ if each $P_{m_{i}}, 1 \leq i \leq n$ has a parametric $\zeta$-set. Hence, if all paths have a parametric unique $\zeta$-set then the spider has same. Hence, $\zeta\left(S_{n}^{*}\right)=1+\sum_{i=1}^{n} \zeta\left(P_{m_{i}}\right)$.

If $n$ copies of $P_{3}$ are connected to $v_{0}$ the $\zeta$-set is unique of order $n$. Therefore the $\zeta$-set is parametric unique. Also $v_{0}$ is not in the $\zeta$-set. However, $P_{3}$ does not have a parametric unique $\zeta$-set. If some of the 3-paths are substituted with paths of the form $P_{m_{i}}, m_{i}=3 j, j=2,3, \ldots$, it follows easily through immediate induction that the $\zeta$-set is parametric unique and does not contain vertex $v_{0}$. Hence, $\zeta\left(S_{n}^{*}\right)=\sum_{i=1}^{n} \zeta\left(P_{m_{i}}\right), m_{i}=3 j, j=1,2,3, \ldots$

For paths $P_{m_{i}}, m_{i}=4+3 j$, or $m_{i}=5+3 j, j=0,1,2 \ldots$ the converse follows implicitly. For paths $P_{m_{i}}$, $m_{i}=3 j, j=1,2,3 \ldots$ the exclusion of $v_{0}$ due to minimization results in a unique choice of a $\zeta$-set with regards to the paths.

Claim 1. For caterpillars $C_{n_{1} \star n_{2} \star, \cdots, n_{k}}, n_{i}, k \geq 1$ a heuristic procedure will be described. This heuristic is adapted from the heuristic described for trees in [5].

Step 1. Let $X_{1}=\left\{v: v \in V\left(P_{m}\right)\right.$, deg $\left.g_{p}(v) \geq 2\right\}$. Delete all $N_{p}[v], v \in X_{1}$ from the caterpillar.

Step 2. Repeat Step 1 exhaustively to obtain sets $X_{2}, X_{3}, \ldots, X_{t}$. This is always possible and yields an explicit disconnected graph consisting of say, $q$ components which could be paths and/or trivial caterpillars.

Step 3. Utilize the heuristic for trees to obtain a $\zeta$-set of each of the $q$ components. Label as sets $Y_{i}, 1 \leq i \leq q$.

Step 4. If each $Y_{i}$ is parametric unique then $\mathcal{C}=\left[\bigcup_{i=1}^{t} X_{i}\right] \cup\left[\bigcup_{j=1}^{q} Y_{j}\right]$ is the parametric unique $\zeta$-set of the caterpillar. Otherwise, the caterpillar does not have a parametric unique $\zeta$-set.

Claim 2. For a lobster $L_{T_{1}, T_{2}, T_{3}, \ldots, T_{\ell}}, T_{i} \in\left\{P_{1}, P_{2}, P_{3}, S_{1, n}\right\}, \ell \geq 1$, a heuristic is described.

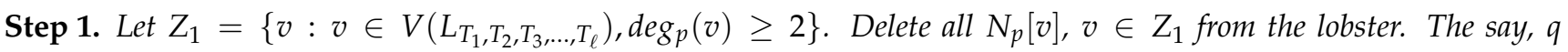
components are path(s) and/or caterpillar(s).

Step 2. Utilize the heuristic for caterpillars to obtain a $\zeta$-set of each of the $q$ components. Label as sets $Y_{i}, 1 \leq i \leq q$.

Step 3. If each $Y_{i}$ is parametric unique then $\mathcal{C}=\left[\bigcup_{i=1}^{t} Z_{i}\right] \cup\left[\bigcup_{j=1}^{q} Y_{j}\right]$ is the parametric unique $\zeta$-set of the lobster. Otherwise, the lobster does not have a parametric unique $\zeta$-set. 
Proposition 5. A finite $(k, d)$-regular tree $T_{k, d}, d \geq 3, k \geq 1$ has a parametric unique $\zeta$-set and,

(a) If $\ell \geq 2$ is even then, $\zeta\left(T_{k, d}\right)=d\left[1+\sum_{t=3,5,7, \ldots,(k-1)}(d-1)^{t-1}\right]$.

(b) If $\ell \geq 3$ is odd then, $\zeta\left(T_{k, d}\right)=1+d\left[\sum_{t=2,4,6, \ldots,(k-1)}(d-1)^{t-1}\right]$.

Proof. It follows easily that for $k=0$ only $v_{0}$ exists. For $\ell \geq 1, d \geq 3$ we have, level $k=1 d$ leafs, level $k=2 d(d-1)$ leafs, $\ldots$, level $k=\ell d(d-1)^{\ell-1}$ leafs.

Case 1. If $\ell \geq 2$ is even then $\mathcal{C}=\underset{k=1,3,5, \ldots,(k-1)}{\bigcup}\{v:$ all $v$ in level $k\}$. It is easy to verify that $\mathcal{C}$ is unique hence, parametric unique. Furthermore, $\zeta\left(T_{k, d}\right)=d\left[1+\sum_{t=3,5,7, \ldots,(k-1)}(d-1)^{t-1}\right]$.

Case 2. If $\ell \geq 3$ is odd then $\mathcal{C}=\left\{v_{0}\right\} \cup \underset{k=2,4,6, \ldots,(k-1)}{\cup}\{v:$ all $v$ in level $k\}$. It is easy to verify that $\mathcal{C}$ is unique hence, parametric unique. Furthermore, $\zeta\left(T_{k, d}\right)=1+d\left[\sum_{t=2,4,6, \ldots,(k-1)}(d-1)^{t-1}\right]$.

\section{Conclusion}

In Corollary 1 the parameter $\kappa\left(C_{n}\right)$ was introduced. From the proof of Proposition 1 it follows directly that for $P_{n}, n=4+3 i, i=0,1,2, \ldots$ we have $\kappa\left(P_{n}\right)=\zeta\left(P_{n}\right)+1=\left\lfloor\frac{n}{3}\right\rfloor+1$. For $P_{n}, n=5+3 i, i=0,1,2, \ldots$ we have $\kappa\left(P_{n}\right)=1$. Furthering research for the parameter $\kappa(G)$ in general remains open. Finding a $\zeta$-set for $G$ in general is known to be NP-complete. Determining isomorphism between graphs is at least in the P-domain.

Conjecture 1. Consider any $\zeta$-set $\mathcal{C}$ of graph $G, \zeta(G) \geq 2$. If for all $\zeta$-sets $\mathcal{C}^{\prime}$ derived from $\mathcal{C}$ such that $\mathcal{C} \cap \mathcal{C}^{\prime} \neq \varnothing$ we have that $\mathcal{C} \cong_{\zeta} \mathcal{C}^{\prime}$ then $G$ has a parametric unique $\zeta$-set.

If the conjecture is proven to be valid it opens an avenue to develop an efficient algorithm to determine parametric uniqueness in a graph. The principles are; (a) determine a $\zeta$-set where-after, (b) find all derivative $\zeta$-sets by substituting say $u \in \mathcal{C}(G)$ with $v \in N_{2}(u)$ and verifying confluence as well as parametric isomorphism. If the condition of confluence and parametric isomorphism are affirmative then parametric uniqueness follows. If the next stronger conjecture holds then the efficiency of an algorithm can be improved significantly.

Conjecture 2. Consider any $\zeta$-set $\mathcal{C}$ of graph $G, \zeta(G) \geq 2$. Let $v \in \mathcal{C}$. If for all $\zeta$-sets $\mathcal{C}^{\prime}$ derived from $\mathcal{C}$ such that $v \in \mathcal{C} \cap \mathcal{C}^{\prime}$, we have that $\mathcal{C} \cong{ }_{\zeta} \mathcal{C}^{\prime}$ then $G$ has a parametric unique $\zeta$-set.

Researching parametric uniqueness with regards to confluence remains open for a vast range of interesting graph families such as circulants, cycle related graphs such as, wheel graphs, helm graphs, sunlet graphs, sun graphs and so on. Other graph parametric sets such as dominating sets, independent sets and others can be studied in respect of parametric uniqueness.

Acknowledgments: The authors would like to thank the anonymous referees for their constructive comments, which helped to improve on the elegance of this paper.

Author Contributions: All authors contributed equally to the writing of this paper. All authors read and approved the final manuscript.

Conflicts of Interest: "The authors declare no conflict of interest."

\section{References}

[1] Bondy, J. A., \& Murty, U. S. R. (1976). Graph Theory with Applications. Macmillan Press, London.

[2] Harary, F. (1969). Graph Theory. Addison-Wesley, Reading MA.

[3] West, B. (1996). Introduction to Graph Theory. Prentice-Hall, Upper Saddle River.

[4] Shiny, J., Kok, J., \& Ajitha, V. Confluence number of graphs. Communicated.

[5] Kok, J., \& Shiny, J. Confluence number of certain derivative graphs. Communicated. 
(C) 2021 by the authors; licensee PSRP, Lahore, Pakistan. This article is an open access article distributed under the terms and conditions of the Creative Commons Attribution (CC-BY) license (http://creativecommons.org/licenses/by/4.0/). 